\title{
Correction to: A comparison of high-throughput plasma NMR protocols for comparative untargeted metabolomics
}

\author{
Nikolaos G. Bliziotis ${ }^{1}$. - Udo F. H. Engelke ${ }^{1}$ - Ruud L. E. G. Aspers ${ }^{2}$ - Jasper Engel ${ }^{2,3}$ - Jaap Deinum ${ }^{4}$. \\ Henri J. L. M. Timmers ${ }^{4} \cdot$ Ron A. Wevers ${ }^{1} \cdot$ Leo A. J. Kluijtmans ${ }^{1}$
}

Published online: 20 May 2020

(c) Springer Science+Business Media, LLC, part of Springer Nature 2020

\section{Correction to: Metabolomics (2020) 16:64 https://doi.org/10.1007/s11306-020-01686-y}

Following publication of the original article, the authors would like to correct a sentence in the paragraph " ${ }^{1} \mathrm{H}-\mathrm{NMR}$ spectra were recorded at $298 \mathrm{~K}$..." under the heading "NMR experiments".

The sentence currently reads:

"The LED pulse sequence had the form -RD-901-G11801-G1-901-G2-T-901-G1-1801-G1-901-G2-t-901-acquire FID, where RD is a relaxation delay, 901 is a $901 \mathrm{RF}$ pulse, G1 is the pulsed-field gradient that is applied to allow editing, 1801 is a $1801 \mathrm{RF}$ pulse, G2 is a spoil gradient applied to remove unwanted magnetization components. The diffusion delay $\mathrm{D}$ is the time during which the molecules are allowed to diffuse-this is the period (901-G1-1801-G1901-G2-T-); and $t$ is a delay to allow the longitudinal eddy currents caused within the sample to decay (Beckonert et al. 2007)."

The sentence should read:

The original article can be found online at https://doi.org/10.1007/ s11306-020-01686-y.

Nikolaos G. Bliziotis

nick.bliziotis@radboudumc.nl

Leo A. J. Kluijtmans

leo.kluijtmans@radboudumc.nl

1 Translational Metabolic Laboratory, Department of Laboratory Medicine, Radboudumc, Geert Grooteplein Zuid 10, 6525 GA Nijmegen, The Netherlands

2 Institute for Molecules and Materials, Radboud University, Houtlaan 4, 6525 XZ Nijmegen, The Netherlands

3 Present Address: Biometris, Wageningen UR, Droevendaalsesteeg 1, 6708 PB Wageningen, The Netherlands

4 Department of Internal Medicine, Radboudumc, Geert Grooteplein Zuid 10, 6525 GA Nijmegen, The Netherlands
"The LED pulse sequence had the form -RD- $90^{\circ}-\mathrm{G} 1$ $180^{\circ}$-G1-90 ${ }^{\circ}$-G2-T- $90^{\circ}$-G1- $180^{\circ}$-G1- $90^{\circ}$-G2- $\tau-90^{\circ}$-acquire FID, where $\mathrm{RD}$ is a relaxation delay, $90^{\circ}$ is a $90^{\circ} \mathrm{RF}$ pulse, G1 is the pulsed-field gradient that is applied to allow editing, $180^{\circ}$ is a $180^{\circ} \mathrm{RF}$ pulse, $\mathrm{G} 2$ is a spoil gradient applied to remove unwanted magnetization components. The diffusion delay $\Delta$ is the time during which the molecules are allowed to diffuse - this is the period $\left(90^{\circ}-\mathrm{G} 1-180^{\circ}-\mathrm{G} 1-90^{\circ}-\mathrm{G} 2\right.$ $\mathrm{T}$-); and $\tau$ is a delay to allow the longitudinal eddy currents caused within the sample to decay (Beckonert et al. 2007)."

This has been corrected with this erratum.

Publisher's Note Springer Nature remains neutral with regard to jurisdictional claims in published maps and institutional affiliations. 